\title{
PRÉSENTER AU LIEU DE REPRÉSENTER UNE ALTERNATIVE À LA QUESTION DE L'INCARNATION SUR SCÈNE?'1
}

\author{
Isabelle Elizéon - Hubert \\ Artiste et chercheuse \\ Doctorante, laboratoire SeféA, IRET, Paris 3
}

Au travers du travail de Robyn Orlin, artiste de la scène originaire d'Afrique du Sud, qui nous pose la question de la norme par la présentation de corps, de figures et de types souvent absents des scènes occidentales, nous explorerons dans cet article la question de l'incarnation sur scène. Nous développerons une réflexion autour d'un nouveau paradigme s'affirmant chaque fois davantage sur la scène contemporaine: le présenter en place du représenter. Nous envisagerons l'acteur, c'est à dire la personne en acte sur scène, en tant que présence provocante de l'humain en place de l'incarnation d'un personnage. Se posera ainsi la problématique suivante: en quoi ce nouveau paradigme, c'est à dire la présence provocante de l'humain au lieu de l'incarnation, la présentation en place de la représentation, peut-il ouvrir à une nouvelle façon de penser, de voir et d'utiliser l'acteur en scène? En quoi peut-il permettre la création de nouveaux imaginaires, que ce soit dans la production d'oeuvres scéniques ou dans la réception du spectateur?

\section{Mots-clés}

Arts de la scène. Théâtre. Performance. Anthropologie. Corps
Através do trabalho de Robyn Orlin, diretora e coreógrafa nativa da Africa do Sul, explorase a questão da encarnação no palco. No seu trabalho artístico, Orlin quebra as normas em vigor pela apresentação de corpos, figuras e tipos ou caras muitas vezes ausentes das cenas ocidentais. Com a exploração deste trabalho será possível desenvolver uma reflexão em torno de um novo paradigma, o qual se afirma cada vez mais na cena contemporânea: o apresentar em vez do representar. Considera-se o ator, ou seja, o "ato no palco", como a presença provocativa do ser humano no lugar da encarnação de um personagem. E assim pergunta-se: como o novo paradigma, ou seja, a presença provocativa do ser humano em vez da encarnação, a apresentação no lugar da representação, pode abrir-se a uma nova maneira de pensar, ver e utilizar o ator no palco? Como pode assim ajudar a criar novos imaginários, seja na produção da obra ou na fase de recepção do espectador?

Palavras-chave

Artes Cênicas. Teatro. Perfomance. Antropologia. Corpo

1 Présenté pour la première fois dans une communication lors de l'Université d'Eté de l'IRET - Paris 3 Sorbonne Nouvelle avec le laboratoire SéFeA, à la Chapelle du Verbe Incarné, pendant le festival d'Avignon, le 15 juillet 2015. 
Au travers du travail de Robyn Orlin', artiste de la scène originaire d'Afrique du Sud, qui nous pose la question de la norme par la présentation de corps, de figures et de types souvent absents des scènes occidentales, nous explorerons dans cet article la question de l'incarnation sur scène. Nous développerons une réflexion autour d'un nouveau paradigme s'affirmant chaque fois davantage sur la scène contemporaine: le présenter en place du représenter. Nous envisagerons l'acteur, c'est à dire la personne en acte sur scène, en tant que présence provocante de l'humain en place de l'incarnation d'un personnage. Se posera ainsi la problématique suivante: en quoi ce nouveau paradigme, c'est à dire la présence provocante de l'humain au lieu de l'incarnation, la présentation en place de la représentation, peut-il ouvrir à une nouvelle façon de penser, de voir et d'utiliser l'acteur en scène? En quoi peut-il permettre la création de nouveaux imaginaires, que ce soit dans la production d'oeuvres scéniques ou dans la réception du spectateur?

En novembre 2014, lors du colloque Rires de Jazz:Esthétique(s) Jazz: la scène et les images $^{2}$ organisé par le laboratoire Séféa, au Musée Dapper, nous avions déjà évoqué le travail de la chorégraphe en proposant le concept de corps-jazz en effervescence ou corps débordant qui permettait d'appréhender le travail de cette artiste sous l'angle du débordement : débordement du cadre traditionnellement assigné à l'acteur, au performer ou au danseur en scène, proposant un dispositif scénique où les corps des artistes et ceux du public entraient dans un jeu relationnel permettant de déplacer et déconstruire les conventions et les stéréo-

\footnotetext{
1 URL: http://www.robynorlin.com/

2 URL: http://www.univ-paris3.fr/rires-de-jazz-esthetique-sjazz-la-scene-et-les-images-2e-edition--292707.kjsp
}

types. Ces derniers étant liés, d'une part, à la question de la représentation spectaculaire, et d'autre part, à la question de la représentation des corps et des identités, notamment dans la figure de l'homme et la femme noir(e)s et des cultures africaines. Cette présente réflexion s'inscrit ainsi dans la suite de cette première analyse.

La question de la présentation en place de la représentation est souvent mise en lien avec le langage performatif. Ce dernier prend en effet de plus en plus de place dans les créations contemporaines répertoriées le plus souvent sous le terme de théâtre postmoderne ou post-dramatique, tel que le définit Patrice Pavis (2014, p. 202). Des artistes, très différents dans leur esthétique, tels Rodrigo Garcia, Pina Bausch, Romeo Castellucci, Jan Lauwers, Bob Wilson ou encore Pippo Delbono partent ou sont partis avant tout de la présence sur le plateau, des histoires ou de l'univers d'acteurs, des danseurs ou performers pour montrer et mettre en scène non pas des personnages mais des présences fortes servant un univers esthétique où domine l'impact visuel. Robyn Orlin, pour sa part, a depuis 1998, avec Daddy, I've seen this piece six times before jusqu'à sa création de 2014 At the same time, we were pointing the finger at you, utilisé le procédé de présentation en place de la représentation.

Afin de clarifier ce que j'entends par présentation, je reprendrai ici les mots de Jean-Frédéric Chevallier, dans son essai Deleuze et le théâtre, rompre avec la représentation : « pour rompre avec la représentation, casser la narration, empêcher l'illustration, libérer la figure : s'en tenir au fait. » (Chevallier, 2015, p.13) Suggérant par là un acte du présenter pour dégager des présences sous la représentation et se diriger vers la découverte du dispars et de 
la singularité qui va rompre avec la représentation de l'identique et de l'attendu. L'importance donnée à la présence de l'artiste en scène, qu'il soit acteur, danseur ou performer, ou tout cela ensemble, rend alors possible un déplacement du regard, une nouvelle perception, exempte d'un discours pré-établi. C'est ce que nous tenterons de démontrer dans cette présente réflexion.

Robyn Orlin joue avec les présences des acteurs/danseurs/performers, tout comme elle joue avec le procédé de la dérision et du cliché. Le jeu combinatoire des présences, de la dérision et du cliché éloigne ainsi le spectateur de l'attendu et de la description. La fragmentation des récits dans les spectacles et la multiplicité des langages utilisés entre performance, théâtralité, danse, vidéo et abolition du 4ème mur, participent également d'une nouvelle réception du spectateur qui se retrouve à regarder dans les images, hors de ces images mais également et à la fois, hors de lui-même. Les artistes en scène se déplacent en permanence sur la frontière floue du jeu, de la représentation d'un personnage, du récit intime ou le paraissant, et de la pure présence. On ne peut alors s'attacher à un personnage ou à une narration. Ce qui est présenté sur scène ne va plus de soi. On navigue plutôt à vue entre les présences présentées là et les événements qui se succèdent. Ce déplacement permanent où le spectateur se retrouve plongé dans un vaet-vient incessant où il n'est pas possible de fixer ou d'appréhender un personnage, va créer un vide où se niche l'innatendu et où pourra se développer la subjectivité du spectateur. Robyn Orlin utilise des codes ou des clichés qui seront démontés successivement pour laisser place à une interrogation, à un malaise ou à une incompréhension, obligeant le spectateur à mettre en place un cheminement intérieur.

Cet entremêlement de significations créé par les acteurs en scène, d'une part, et, d'autre part, créé par la perception du public permet d'engendrer un espace composé par les singularités de chacun : singularités de la chorégraphe et metteuse en scène, singularités des acteurs/danseurs/performers, singularités enfin de chaque personne du public. Présenter en place de, ou en simultanéité de représenter/ incarner un personnage en scène, c'est placer ainsi la logique du ET (E-T) en place du OU. Et revendiquer une logique de I'ENTRE-DEUX. L'acteur en scène n'est pas ce personnage OU cet autre. II est tout cela et autre chose, toujours entre deux mouvements, deux identités, deux actions, deux caractères. II ne se définit pas par ce qu'il semble être ou représenter mais par ce qu'il fait, par ce qui le meut : danseuse classique affublée de chaussons de danse aux mains et aux pieds tentant de faire le mouton (L'Allegro il penseroso ed il moderato" de Haendel, à l'Opéra Garnier), autre femme en robe et lunettes glissant sur des assiettes en plastiques rouges (Daddy, I've seen this piece six times before), homme élégant coiffé d'un sac rouge discutant avec le public de la possibilité de trouver un bon manager pour ses spectacles (Coupé-Décalé, criado com James Carlès). L'importance des actions des acteurs/ performers et des interactions avec le public dans les spectacles de Orlin les mettent en lien direct avec ce que le metteur en scène Jérôme Bel5 explique, dans sa correspondance avec Boris Charmatz : «Qu'est-ce qu'un spectacle ? [...] le spectacle consiste à faire des choses pas à les raconter »(Bell; Charmatz, 2013, p.73).

Tous ces acteurs/danseurs/performers sont donc là pour faire, montrer, présenter 
quelque chose. Ils sont avant tout présences et non personnages. Et si, dans le cas des spectacles de Robyn Orlin, ils peuvent initialement sembler représenter quelque chose, très vite la figure s'évanouira et le spectateur, gêné dans sa représentation première, devra se diriger vers d'autres perceptions, d'autres constructions. Jean-Frédéric Chevallier citant encore Deleuze explique : « la pensée contemporaine « naît de la faillite de la représentation, comme de la perte des identités, et de la découverte de toutes les forces qui agissent sous la représentation de l'identique » (2015, p.19). Chevallier insiste sur la relation entre idéologie et représentation. C'est sans doute sur ce point que penser et pratiquer le théâtre comme art du présenter peut prendre toute son importance. Robyn Orlin dans ses créations participe de cette déconstruction des idéologies, quelles soient post-coloniales, mercantiles ou tout simplement théâtrales. Ses spectacles œuvrent ainsi non pas pour une représentation mais pour une expérimentation.

Ces notions d'inattendu et d'expérimentation se retrouvent également dans le travail de certains dramaturges contemporains issus de la diaspora africaine, comme Robyn Orlin. Kossi Efoui, dans le recueil de Sylvie Chalaye, Le Syndrôme Frankestein (2004, p. 24), le formule ainsi: “ il s'agit de ne plus être présent là où on est attendu, mais systématiquement donner rendez-vous ailleurs, déplacer les questions ailleurs ». Kossi Efoui reprend un peu plus loin, en ces termes: "La fameuse rupture est idéologique: être en dehors. Et être en dehors c'est être en dehors d'un discours dominant ». Koffi Kwahulé, dramaturge ivoirien, défend, quand à lui, une opacité dans son écriture, un empêchement de tout rapport discursif immédiat. Marcel Zang, pour sa part, parle de l'inconnu comme le moyen d'entrer en mouvement, de retrouver la vie, le rythme, la turbulence. II dit encore: « tout ce qui est variable, tout ce qui bouge, qui évolue, dérange forcément. La meilleure façon de ne pas être dérangé c'est d'arrêter le mouvement de l'autre »(Chalaye, 2004, p. 65). Robyn Orlin, enfin, déclare: «Rien n'est immuable. Rien n'est jamais ce qu'il paraît être, ni ce que l'on pense qu'il est » (Hespel, p. 49).

II est notable qu'aujourd'hui, en cette première décennie du 21ème siècle, l'immigré, l'exilé, l'errant urbain, et même le touriste sont des figures dominantes de nos cultures contemporaines. L'individu aujourd'hui évoque, comme le développe Nicolas Bourriaud dans son essai Radicant - pour une esthétique de la globalisation (2009), ces plantes qui ne s'en remettent pas à une racine unique pour croître mais progressent en tout sens sur les surfaces qui s'offrent à elles. Le théâtre du présenter pourrait ainsi se définir de même, par un processus de sélections, d'ajouts, de multiplications. II organise les signes afin de multiplier une identité par une autre. Les acteurs en scène dans ce théâtre du présenter ont des enracinements successifs, simultanés ou croisés. Ils se matérialisent et s'actualisent dans une logique inclusive du ET et non du OU, comme évoqué plus haut. Ils préfèrent à toute incarnation le jeu mouvant des panoplies successives. Ils revendiquent, se faisant, une apologie de I'hétérogénéité et de la pluralité des mondes. Ils sortent de l'enclos et de l'assignation à une identité. Ils renvoient, comme l'évoque Michel Maffesoli, à une identité en mouvement, une identité fragile, qui n'est plus, comme ce fut le cas dans la modernité, le seul fondement solide de l'existence individuelle et sociale.

Au travers de cette pensée en action, 
dans les spectacles de Robyn Orlin, mais aussi dans ceux des dramaturges cités plus haut, se dessine un positionnement politique où est questionné le rapport des gens entre eux et leur rapport au monde, essayant de détourner, de déplacer le discours dominant. Développer la pensée et la pratique d'un théâtre du présenter, c'est ainsi oeuvrer pour une ouverture et un déplacement des identités, quelles soient sociales, historiques, géographiques ou sexuelles. C'est, comme nous l'évoquions plus haut, créer de l'inattendu et revendiquer l'expérimentation, la participation active du spectateur en lui ouvrant la possibilité d'un nouveau regard sur le monde, les mondes.

Dans ce nouveau paradigme du présenter au théâtre, le spectateur n'est plus face au consensus de la représentation qui demande un allant-de-soi dans la réception, un code commun, unique et préexistant. II entre dans un cheminement où son imaginaire va devoir se mettre en branle, là où ne seront posées que des questions, là où ne seront données que des ouvertures, des possibles. Les acteurs ainsi présents sur scène sont des individus en perpétuel devenir, des êtres en mouvement. Ils présentent des figures puis les défont, ils présentent des images et les effacent, ils sont dans le faire, dans l'immédiateté de l'action. Ce théâtre là, dans la création continue de mouvements et d'interstices, rend propice aux écarts et, par là, donne au spectateur le pouvoir d'être producteur de sa propre vision, de son propre questionnement et donc de se mouvoir à son tour. Le théâtre du présenter serait alors un théâtre de l'ouverture et du déplacement, où dans l'insatisfaction des formules et des catégories pré-existantes, on tenterait de se frayer de nouveaux chemins en mettant en route nos racines tout en leur déniant, com- me le dit Nicolas Bourriaud, la vertu de définir complètement notre identité. Dans le théâtre du présenter, l'acteur en scène tout comme le spectateur deviennent des Homo-viator, dont les passages à travers les formes, les actions et les signes renvoient à l'expérience du déplacement et de la traversée, nécessitant une perpétuelle traduction de la part du spectateur, une remise en question de ses acquis et une mise en chemin de son imaginaire.

Referências

BEL, Jérôme; CHARMATZ, Boris. Emails 20092010. Paris: Les Presses du Réel, 2013.

BOURRIAUD, Nicolas. Radicant - pour une esthétique de la globalisation. Paris: Denoël, 2009.

CHALAYE, Sylvie. Afrique noire et dramaturgies contemporaines: le Syndrôme Frankestein. Paris: Éditions Théâtrales, 2004.

CHEVALLIER Jean-Frédéric. Deleuze et le théâtre - rompre avec la représentation. Besançon: Les Solitaires Intempestifs, 2015.

HESPEL, Olivier. Robyn Orlin - fantaisiste rebelle. Toulouse/Pantin: Éditions de l'Attribut/ Centre National de la Danse, 2007.

PAVIS, Patrice. Dictionnaire de la performance et du théâtre contemporain. Paris: Armand Colin, 2014.

Recebido em: 28/09/2016 Aprovado em: 18/10/2016 